Optimal portfolio selection for general provisioning and terminal wealth problems

Koen Van Weert; Jan Dhaene; Marc Goovaerts

DEPARTMENT OF ACCOUNTANCY, FINANCE AND INSURANCE (AFI) 


\title{
Optimal Portfolio Selection for General Provisioning and Terminal Wealth Problems
}

\author{
Koen Van Weert ${ }^{*, \dagger} \quad$ Jan Dhaene ${ }^{\dagger, \ddagger} \quad$ Marc Goovaerts ${ }^{\dagger, \ddagger}$ \\ ${ }^{\dagger}$ K.U.Leuven, Dept. of Accountancy, Finance and Insurance, \\ Naamsestraat 69, B-3000 Leuven, Belgium. \\ ${ }^{\ddagger}$ University of Amsterdam, Dept. of Quantitative Economics, \\ Roetersstraat 11, 1018 WB Amsterdam, The Netherlands. \\ ${ }^{*}$ Corresponding author: koen.vanweert@econ.kuleuven.be.
}

Version: October 8, 2009

\begin{abstract}
In Dhaene et al. (2005), multiperiod portfolio selection problems are discussed, using an analytical approach to find optimal constant mix investment strategies in a provisioning or savings context. In this paper we extend some of these results, investigating some specific, real-life situations. The problems that we consider in the first section of this paper are general in the sense that they allow for liabilities that can be both positive or negative, as opposed to Dhaene et al. (2005), where all liabilities have to be of the same sign. Secondly, we generalize portfolio selection problems to the case where a minimal return requirement is imposed. We derive an intuitive formula that can be used in provisioning and terminal wealth problems as a constraint on the admissable investment portfolios, in order to guarantee a minimal annualized return. We always apply our results to optimal portfolio selection.
\end{abstract}

\section{Introduction}

In Dhaene et al. (2005), multiperiod portfolio selection problems are discussed, using an analytical approach to find optimal constant mix investment strategies in a provisioning or savings context. In this paper we extend some of these results, investigating some specific, real-life situations.

Determining the distribution function of a sum of random variables, describing a series of future payments, is important when solving several problems in a general insurance or finance context. In Dhaene et al. (2005), solutions are provided, both in a provisioning and 
saving context, in case all payments are of the same sign. In Vanduffel et al. (2005a), a socalled saving-consumption plan is considered, where a series of positive payments (savings) is followed by a series of negative ones (consumptions). In this paper we extend the solution of Vanduffel et al. (2005a) to the more general contexts of provisioning and saving as described in Dhaene et al. (2005), allowing for more arbitrary cash flows patterns. We show that allowing cash flows of mixed signs does not necessarily mean that comonotonic approximations can not be used anymore. As a result we significantly expand the scope of problems for which the distribution function can be accurately approximated using comonotonic approximations. A lot of practical situations exist where sporadic negative payments occur. For instance, when determining the liabilities of a pension fund, outgoing and incoming cash flows are typically compared. It could easily happen that in some years the incoming cash flows are larger than the outgoing, leading to negative liabilities in these years.

Secondly, we generalize portfolio selection problems to the case where a minimal return requirement is imposed. We derive an intuitive formula that can be used as a constraint on the admissable investment portfolios, in order to guarantee a minimal annualized return, with a related probability level over a specified time period. This formula can be used in provisionig as well as terminal wealth problems.

We always apply our results to optimal portfolio selection problems, and illustrate with numerical examples. In the following section a short description is given of the framework of optimal portfolio selection in which we work. For more details and practical examples we refer to Dhaene et al. (2005).

\subsection{Lognormal Framework and Optimal Portfolio Selection}

When discussing optimal portfolio selection problems, we assume throughout this paper the classical continuous-time framework of Merton (1971), also known as the Black \& Scholes (1973) setting. We suppose there are $m$ risky assets available. We restrict to constant mix strategies: the fractions invested in the different assets remain constant over time. We denote the vector describing the portfolio process as $\underline{\pi}^{T}=\left(\pi_{1}, \ldots, \pi_{m}\right)$, where $\pi_{i}$ is the proportion invested in risky asset $i$, with $\sum_{i=1}^{m} \pi_{i}=1$. In our examples we assume there is no risk-free asset class available. Although our results also hold in the general case, we assume short-selling is not allowed, which means $0 \leq \pi_{i} \leq 1$ for all $i$. See e.g. Björk (1998) for more details on the Black \& Scholes setting. Throughout this paper we use the same notations and terminology as in Dhaene et al. (2005).

As both the time period and the investment horizon that we consider are typically long, the use of a Gaussian model for the stochastic returns can be justified by Central Limit Theorem arguments, see e.g. Cesari \& Cremonini (2003) and Levy (2004) for some empirical evidence.

Investing an amount of 1 at time $k-1$ in asset $i$ will grow to $e^{Y_{k}^{i}}$ at time $k$. For a fixed asset $i$, the random variables $Y_{k}^{i}$ are assumed i.i.d., normally distributed with mean $\mu_{i}-\frac{1}{2} \sigma_{i}^{2}$ and variance $\sigma_{i}^{2}$. 
We denote the drift vector and the variance-covariance matrix of the risky assets by $\underline{\mu}^{T}=\left(\mu_{1}, \ldots, \mu_{m}\right)$, and $\underline{\Sigma}$ respectively. The drift vector and volatility corresponding to an investment portfolio $\underline{\pi}$ are written as $\mu(\underline{\pi})$ and $\sigma^{2}(\underline{\pi})$. We have that

$$
\mu(\underline{\pi})=\underline{\pi}^{T} \underline{\mu} \text { and } \sigma^{2}(\underline{\pi})=\underline{\pi}^{T} \cdot \underline{\Sigma} \cdot \underline{\pi} .
$$

The yearly returns $Y_{i}(\underline{\pi})$ of an investment portfolio $\underline{\pi}$ are independent and normally distributed random variables, with expected value $\mathrm{E}\left[Y_{i}(\underline{\pi})\right]=\mu(\underline{\pi})-\frac{1}{2} \sigma^{2}(\underline{\pi})$ and variance $\operatorname{Var}\left[Y_{i}(\underline{\pi})\right]=\sigma^{2}(\underline{\pi})$.

When no confusion is possible, we omit the dependence on the investment portfolio $\underline{\pi}$ in the notations. Hence the yearly returns are modelled by the i.i.d., normally distributed random variables $Y_{i}$, with mean $\mu-\frac{1}{2} \sigma^{2}$ and standard deviation $\sigma$.

\section{Comonotonic Approximations in case of Cash-Flows of Mixed Signs}

\subsection{Problem description}

Consider the sum

$$
S=\sum_{i=0}^{n} \alpha_{i} e^{Z_{i}}
$$

where the $\alpha_{i}$ are deterministic constants, and the $Z_{i}$ are linear combinations of the components of the multivariate normal random vector $\left(Y_{1}, Y_{2}, \ldots, Y_{n}\right)$ :

$$
Z_{i}=\sum_{j=1}^{n} \lambda_{i j} Y_{j} .
$$

As the random variable $S$ is a sum of non-independent lognormal variables, it is impossible to determine the distribution function of $S$ analytically. Several approximation techniques have been proposed throughout the literature, see e.g. Asmussen \& Rojas (2005), Dufresne (2004), Milevsky \& Posner (1998) and Milevsky \& Robinson (2000). In this paper we will use convex upper and lower bounds based on comonotonicity, see e.g. Kaas et al. (2000). See also Huang et al. (2004) or Vanduffel et al. (2005b) for a comparison of some of these approximation techniques.

In Dhaene et al. (2005) the following bounds for $S$ are derived:

$$
S^{c}=\sum_{i=0}^{n} \alpha_{i} e^{E\left[Z_{i}\right]+\sigma_{Z_{i}} \Phi^{-1}(U)}
$$

and

$$
S^{l}=E[S \mid \Lambda]=\sum_{i=0}^{n} \alpha_{i} e^{E\left[Z_{i}\right]+\frac{1}{2}\left(1-r_{i}^{2}\right) \sigma_{Z_{i}}^{2}+r_{i} \sigma_{Z_{i}} \Phi^{-1}(U)},
$$


with $U$ uniformly distributed on the unit interval, $\Phi$ the standard normal cdf, $r_{i}$ the correlations between the random variables $Y_{i}$ and

$$
\Lambda=\sum_{j=1}^{n} \beta_{j} Y_{j}
$$

$S^{l}$ and $S^{c}$ are respectively a lower and upper bound for $S$ in convex order:

$$
S^{l} \leq_{c x} S \leq_{c x} S^{c}
$$

For more details on e.g. the correlation coefficients $r_{i}$ and the choice of the coefficients $\beta_{j}$ in (6) we refer to Dhaene et al. (2005). For more details about these approximations, its relation with the concept of comonotonicity and its applications in insurance and finance, see e.g. Dhaene et al. (2002 a,b). For more details on ordering of random variables see e.g. Kaas et al. (2008).

If all the amounts $\alpha_{i}$ are of the same sign, (4) and (5) are comonotonic sums, which implies that distortion risk measures related to these bounds can be obtained by simply summing the individual terms in the sum. However, in case of payments $\alpha_{i}$ with changing signs, $S^{l}$ and $S^{c}$ are not necessarily comonotonic sums. The upper bound approximation (4) can be adapted easily as follows:

$$
S^{c}=\sum_{i=0}^{n} \alpha_{i} e^{E\left[Z_{i}\right]+\operatorname{sign}\left(\alpha_{i}\right) \sigma_{Z_{i}} \Phi^{-1}(U)},
$$

with $\operatorname{sign}(x)=1$ for $x>0$, and $\operatorname{sign}(x)=-1$ for $x<0$ (see e.g. Dhaene et al. (2002a,b)). We have that (8) is a comonotonic sum. However, the upper bound does in general not give a very accurate approximation of the distribution function of $S$, the accuracy of the lower bound (5) is usually much higher. For this lower bound, the problem is that it is not possible to find a conditioning random variable $\Lambda$, leading to an accurate approximation of $S$, such that $S^{l}$ is a sum of non-decreasing functions of $\Lambda$ (and hence such that $S^{l}$ is a comonotonic sum) in case the $\alpha_{i}$ 's have changing signs. As mentioned above, this would mean that distortion risk measures related to $S^{l}$ can not be obtained by simply summing the individual terms in the sum, which would make the lower bound approximations useless in practice.

In this paper however we show that it is possible, under some mild conditions, to allow for more arbitrary cash flows patterns. We show that allowing some of the cash flows to be negative does not necessarily imply comonotonic lower bound approximations can not be used. As a result we significantly expand the scope of problems and cash flow patterns for which the distribution function can be accurately approximated. A lot of practical situations exist where sporadic negative payments occur. For instance, when determining the liabilities of a pension fund, outgoing and incoming cash flows are typically compared. It may happen that in some years the incoming cash flows are larger than the outgoing ones, leading to negative liabilities in these years.

In Section 2.2 we look at the problem of allowing negative savings (or withdrawals) when using a comonotonic lower bound approximation in a saving and terminal wealth 
context. We generalize the problem described in Vanduffel et al. (2005a), where a so-called 'saving-consumption' plan is considered, in which a series of positive payments (savings) is followed by a series of negative ones (consumptions). In Section 2.3 we discuss allowing negative liabilities (hence contributions) in a context of reserving. In both sections we work in the lognormal framework as described in Section 1.1.

\subsection{Saving and terminal wealth}

In this section, we consider the problem of how to invest periodic amounts in order to reach some target capital at a predetermined future time $n$. Consider a set of deterministic amounts $\alpha_{0}, \alpha_{1}, \cdots, \alpha_{n-1}$ with $n \geq 1$. It will follow from our main result that $\alpha_{0}$ has to be positive. The amounts $\alpha_{i}$ with $0<i<n$ can take any value, positive or negative. This is a generalization of the problem described in Vanduffel et al. (2005a), where a so-called 'saving-consumption' plan is considered, in which a series of positive payments (savings) is followed by a series of negative ones (consumptions).

Notations and terminology used in this section are based on Vanduffel et al. (2005a). We assume the return on the account is generated by a geometric Brownian motion process, as explained in Section 1.1. Let $V_{k}$ denote the surplus at time $k$. By convention, the surplus at time $k$ has to be understood as the surplus just after saving or withdrawal. Starting from the initial value $V_{0}=\alpha_{0}$, the surplus $V_{k}$ available at time $k$ is given by the following recursive relation:

$$
V_{k}=V_{k-1} e^{Y_{k}}+\alpha_{k}, \quad k=1, \cdots, n-1 .
$$

The surplus at time $n$ is then equal to $V_{n}=V_{n-1} e^{Y_{n-1}}$. Solving recursion (9), we can rewrite $V_{k}$ in the form of (2) as

$$
V_{k}=\sum_{i=0}^{k} \alpha_{i} e^{Z_{i}}, \quad k=0, \cdots, n-1,
$$

with $Z_{i}=\sum_{j=i+1}^{k} Y_{j}$, for $i=0, \cdots, k$. By convention $\sum_{j=k+1}^{k} Y_{j}=0$. The surplus at time $n$ equals

$$
V_{n}=\sum_{i=0}^{n-1} \alpha_{i} e^{Z_{i}}, \quad \text { with } Z_{i}=\sum_{j=i+1}^{n} Y_{j} .
$$

Note that this surplus can become negative, which would imply shortselling of units of the investment account. Our goal is to describe the distribution of the final surplus $V_{n}$. To avoid allowing for shortselling we only look at the distribution of the final wealth $W_{n}$, which we define as:

$$
W_{n}=\max \left[V_{n}, 0\right], \quad k=0, \ldots, n .
$$

As explained in the previous section, we focus on the comonotonic lower bound (5), which we denote here as $V_{n}^{l}$. We approximate the distribution of the final wealth $W_{n}$ by $W_{n}^{l}=\max \left[V_{n}^{l}, 0\right]$. 
Choosing $\Lambda$ such that the variance of $V_{n}^{l}$ is maximized, and hence as close as possible to $\operatorname{Var}\left[V_{n}\right]$, results in the optimal conditioning random variable $\Lambda$ of the form (6), with coefficients $\beta_{j}$ equal to:

$$
\beta_{j}=\sum_{i=0}^{j-1} \alpha_{i} e^{(n-i) \mu}
$$

for $j=1, \ldots, n$, with $\mu$ the drift of the yearly return $Y_{j}$, as explained in Section 1.1. The procedure to determine this optimal $\Lambda$ is explained in detail in Vanduffel et al. (2005a).

From (5) we find that the random variable $V_{n}^{l}$ with $\Lambda$ chosen as (6) with coefficients (13) is distributed as

$$
V_{n}^{l} \stackrel{d}{=} \sum_{i=0}^{n-1} \alpha_{i} e^{(n-i) \mu-\frac{1}{2} r_{i}^{2}(n-i) \sigma^{2}+r_{i} \sigma \sqrt{n-i} \Phi^{-1}(U)},
$$

where $\stackrel{d}{=}$ stands for 'equality in distribution', $U$ is uniformly distributed on $(0,1)$, and the coefficients $r_{i}$ are given by

$$
r_{i}=\frac{\operatorname{cov}\left(Z_{i}, \Lambda\right)}{\sigma_{Z_{i}} \sigma_{\Lambda}}=\frac{\sum_{j=i+1}^{n} \beta_{j}}{\sqrt{n-i} \sqrt{\sum_{j=1}^{n} \beta_{j}^{2}}}, \quad i=0, \cdots, n-1
$$

and $r_{n}=0$.

Throughout the remainder of this section we use the notation $f$ for the following function

$$
f(p)=\sum_{i=0}^{n-1} \alpha_{i} e^{(n-i) \mu-\frac{1}{2} r_{i}^{2}(n-i) \sigma^{2}+r_{i} \sigma \sqrt{n-i} \Phi^{-1}(p)},
$$

for $p \in(0,1)$. Combining (12) and (14) we see that $V_{n}^{l} \stackrel{d}{=} f(U)$ and $W_{n}^{l} \stackrel{d}{=} \max [f(U), 0]$.

In order to proof the main result of this section, we state the following lemma:

Lemma 1 Let $f$ be defined by (16) and $\beta_{j}$ by (13). If $\beta_{j}>0$ for $j=1,2, \ldots, n$, then for any $p$ in the unit interval $(0,1), f(p) \geq 0$ implies $f^{\prime}(p)>0$.

See Vanduffel et al. (2005a) for a proof of this Lemma.

The main result of this section is stated in the following Theorem.

Theorem 1 If the conditioning random variable $\Lambda$ is chosen as (6) with coefficients (13), and if

$$
E\left[V_{j}\right]>0, \quad j=0, \cdots, n-1,
$$

then the quantiles of $W_{n}^{l}$ are given by

$$
Q_{p}\left[W_{n}^{l}\right]=\max [f(p), 0] \quad 0<p<1,
$$

whereas the distribution function of $W_{n}^{l}$ follows from

$$
f\left(F_{W_{n}^{l}}(x)\right)=x, \quad x \geq 0,
$$

with $f(p)$ defined by (16). 
Proof. It follows from (13) that for $j=1,2, \ldots, n$,

$$
\beta_{j}=e^{n-j+1} \sum_{i=0}^{j-1} \alpha_{i} e^{(j-1-i) \mu}=e^{n-j+1} \mathrm{E}\left[V_{j-1}\right] .
$$

In other words, condition (17) implies that $\beta_{j}>0$ for $j=1,2, \ldots, n$. Since all $\beta_{j}$ are strictly positive, we find from Lemma 1 that the function $\max [f(p), 0]$ is non-decreasing (and continuous) on the interval $(0,1)$. As stated in Vanduffel et al. (2005a), the quantiles of $W_{n}^{l}$ can easily be determined analytically in this case:

$$
Q_{p}\left[W_{n}^{l}\right]=\max [f(p), 0], \quad p \in(0,1) .
$$

Vanduffel et al. (2005a) also show that, under the conditions of Lemma 1, the d.f. of $W_{n}^{l}$ can be determined from

$$
f\left(F_{W_{n}^{l}}(x)\right)=x, \quad x \geq 0
$$

which completes the proof.

It is clear that any reasonable plan should fulfill condition (17), which states that the average surplus $\mathrm{E}\left[V_{j}\right]$ should be non-negative at any time. Note that for $j=0$, condition (17) can be rewritten as $\alpha_{0}>0$.

For given cash flows $\alpha_{i}, i=1, \ldots, n-1$, we have that $\mathrm{E}\left[V_{i}\right]=\sum_{j=0}^{i} \alpha_{j} e^{(i-j) \mu}$ is an increasing function of $\mu$. In other words, for given cash flows $\alpha_{i}$, conditions (17) can be rewritten as a single condition on $\mu$ as follows:

$$
\mu>\mu^{*}=\max \left(\min \left\{\mu \mid \sum_{j=0}^{i} \alpha_{j} e^{(i-j) \mu}>0 ; i=1, \ldots, n-1\right\}, 0\right)
$$

Theorem 1 is a generalization of the main result of Vanduffel et al. (2005a), which stated that in case of a 'saving-consumption' plan the average final surplus had to be non-negative for (18) and (19) to hold.

Our result shows that allowing some of the cash flows to be negative does not necessarily mean that comonotonic lower bound approximations can not be used. As explained in the previous section, this result significantly expands the scope of problems and cash flow patterns for which the distribution function of the final wealth can be accurately approximated. An example is the situation where you have a relatively small fixed income (e.g. yearly), and relatively large periodical expenses (e.g. every 5 years). This particular situation is studied in the next paragraph.

\subsubsection{Special case: constant savings and consumptions}

In this section we consider a deterministic cash flow stream where

$$
\alpha_{i}=\left\{\begin{array}{ll}
\alpha-1 & \text { if } i=j m, j=1, \ldots, k \\
\alpha & \text { otherwise, } 0 \leq i<n .
\end{array}, \text { with } k m=n-1 .\right.
$$


We have a fixed yearly income $\alpha$, for some $\alpha>0$, for a period of $n$ years. Furthermore we have fixed liabilities: a fixed amount 1 is to be paid every $m$ years (with $m>1$ ).

To start we investigate when conditions (17) are satisfied in this special case. First note that the conditions are fulfilled in case $\mathrm{E}\left[V_{i m}\right]>0$ for $i=0, \ldots, k$, as these are the only years in which a negative cash flow is involved. Condition (17) for $\mathrm{E}\left[V_{i m}\right]$ can be rewritten as:

$$
\mathrm{E}\left[V_{i m}\right]>0 \Leftrightarrow \alpha>e^{-m \mu} \frac{1-e^{-i m \mu}}{1-e^{-m \mu}} \frac{e^{-\mu}-1}{e^{-(i m+1) \mu}-1}
$$

As the right-hand side of the latter inequality is increasing in $i$, conditions (17) hold in case $\mathrm{E}\left[V_{k m}\right]=\mathrm{E}\left[V_{n-1}\right]>0$, or, in terms of $\alpha$ :

$$
\alpha>\alpha^{*}=e^{-m \mu} \frac{1-e^{-(n-1) \mu}}{1-e^{-m \mu}} \frac{e^{-\mu}-1}{e^{-n \mu}-1} .
$$

Example 1 Suppose we have to pay an amount of 1 every 5 years over a period of 25 years (or $m=k=5, n=26$ ). Also suppose $\mu=0.07$. Condition (26) indicates we can apply the results from Theorem 1 if we have a yearly income $\alpha$ bigger than $\alpha^{*}=0.1591$.

If condition (26) is satisfied, we find from Theorem 1 that the approximated quantiles $Q_{p}^{+}\left[W_{n}^{l}\right]$ and the approximated probabilities $F_{W_{n}^{l}}(x)$ follow from (18) and (19) with $f(p) \equiv$ $f_{\alpha}(p)$ given by

$$
f_{\alpha}(p)=\alpha \sum_{i=0}^{n-1} e^{(n-i) \mu-\frac{1}{2} r_{i}^{2} \sigma_{Z_{i}}^{2}+r_{i} \sigma_{Z_{i}} \Phi^{-1}(p)}-\sum_{i=1}^{k} e^{(n-i m) \mu-\frac{1}{2} r_{i m}^{2} \sigma_{Z_{i m}}^{2}+r_{i m} \sigma_{Z_{i m}} \Phi^{-1}(p)} .
$$

Let $W_{n}(\alpha, k, m)$ and $W_{n}^{l}(\alpha, k, m)$ denote the (approximated) final wealth for the aforementioned plan, with a yearly income of $\alpha$, and a liability of 1 every $m$ years over a period of $k m=n-1$ years.

Next, we determine the minimal yearly income $\alpha$ such that the probability of a shortfall, $F_{W_{n}(\alpha)}(0)$, is at most equal to a certain $\epsilon$. In other words, we determine $\alpha(\epsilon)$ as

$$
\alpha(\epsilon)=\inf \left\{\alpha \mid F_{W_{n}(\alpha, k, m)}(0) \leq \epsilon\right\} .
$$

Since $F_{W_{n}(\alpha, k, m)}(0)$ is strictly decreasing and continuous in $\alpha, \alpha(\epsilon)$ follows from the equation

$$
F_{W_{n}(\alpha(\epsilon), k, m)}(0)=\epsilon .
$$

This $\alpha(\epsilon)$ can be approximated by $\alpha^{l}(\epsilon)$, which can be determined by solving

$$
F_{W_{n}^{l}\left(\alpha^{l}(\epsilon), k, m\right)}(0)=\epsilon .
$$

As stated before, we can apply the results of Theorem 1 in case $\epsilon$ is such that $\alpha^{l}(\epsilon)>\alpha^{*}$. Hence, it follows from (19) and (30) that the approximated minimal savings effort $\alpha^{l}(\epsilon)$ can be found from

$$
f_{\alpha^{l}(\epsilon)}(\epsilon)=0 \quad \text { if } \alpha^{l}(\epsilon)>\alpha^{*} .
$$


Numerical illustration Assume the yearly returns $Y_{i}$ have expectation and variance given by $\mu-\frac{\sigma^{2}}{2}$ and $\sigma^{2}$, with $\mu=0.07$ and $\sigma=0.15$. As in Example 1, consider a plan with a fixed liability of 1 every 5 years over a period of 25 years $(m=k=5$, and $n=26)$. As seen in Example 1, condition (26) is equivalent to $\alpha>0.1591$. Using (19), we can approximate the probability of shortfall $\operatorname{Pr}\left[W_{26}^{l}=0\right]$. In Table 1 this probability is given for a range of yearly incomes $\alpha$.

Using (31), we can also determine the minimal yearly income $\alpha$ such that the probability of shortfall is less than a given $\epsilon$. For instance, the approximated minimal yearly income $\alpha^{l}(0.05)$, which guarantees that the probability of shortfall is less than or equal to $5 \%$, is equal to 0.1910 . Or, suppose we want a yearly survival probability of $99.5 \%$, which corresponds to a long term survival probability of approximately $0.995^{25}=0.8822$. In this case we find a minimal yearly income $\alpha^{l}(0.1178)$ equal to 0.1845 .

\begin{tabular}{c|c}
$\alpha$ & $\operatorname{Pr}\left[W_{26}^{l}=0\right]$ \\
\hline 0.1591 & $63.72 \%$ \\
0.1600 & $61.94 \%$ \\
0.1700 & $40.18 \%$ \\
0.1800 & $18.81 \%$ \\
0.1900 & $5.85 \%$ \\
0.2000 & $1.19 \%$
\end{tabular}

Table 1: Approximated probability of shortfall

\begin{tabular}{c|c|cc}
$p$ & $Q_{p}\left[W_{26}^{l}\right]$ & $' Q_{p}\left[W_{26}\right] '$ & s.e. \\
\hline 0.99 & 13.0510 & 13.1035 & 0.18 \\
0.95 & 7.5174 & 7.5196 & 0.09 \\
0.90 & 5.5375 & 5.5337 & 0.03 \\
0.75 & 3.2299 & 3.2315 & 0.03 \\
0.50 & 1.6520 & 1.6602 & 0.02 \\
0.25 & 0.7142 & 0.7191 & 0.01 \\
0.10 & 0.2051 & 0.1914 & 0.01 \\
0.05 & 0 & 0 & 0.00
\end{tabular}

Table 2: Approximated and simulated values for the quantiles of $W_{25}$

To illustrate the accuracy of the lower bound approximation $W_{26}^{l}$, we compare the approximated quantiles $Q_{p}\left[W_{26}^{l}\right]$ (calculated using (21)) with the simulated quantiles ' $Q_{p}\left[W_{26}\right]$ '. We compute the quantiles for the optimal strategy obtained earlier, with $\alpha=\alpha^{l}(0.05)=$ 0.1910 . The results are given in Table 2 . We see for instance that there is a $10 \%$ probability that the final wealth at time 25 will exceed 5.5337 (simulated value). The approximated value for this final wealth is equal to 5.5375 . 


\subsubsection{Application to Optimal Portfolio Selection}

To apply the results of Section 2 to optimal portfolio selection problems, we slightly adapt notations. The surplus $V_{k}$ (see (10)) and wealth $W_{k}$ (see (12)) are in this setting a function of an investment portfolio $\underline{\pi}$, which we denote as:

$$
V_{k}(\underline{\pi})=\sum_{i=0}^{k} \alpha_{i} e^{Z_{j}(\underline{\pi})}=\sum_{i=0}^{k} \alpha_{i} e^{\sum_{i=j+1}^{k} Y_{i}(\underline{\pi})}, \quad k=0, \ldots, n-1,
$$

with

$$
V_{n}(\underline{\pi})=V_{n-1}(\underline{\pi}) e^{Y_{n}(\underline{\pi})},
$$

and

$$
W_{k}(\underline{\pi})=\max \left[V_{k}(\underline{\pi}), 0\right], \quad k=0, \ldots, n .
$$

As follows from Theorem 1, we should only look at the portfolios $\pi$ for which conditions (17), or, equivalently, condition (23), is fulfilled. In other words, we have to restrict any optimization to the following set of admissable portfolios $\Theta$ :

$$
\Theta=\left\{\underline{\pi} \mid \mathrm{E}\left[V_{i}(\underline{\pi})\right]>0 ; i=0, \ldots, n-1\right\}=\left\{\underline{\pi} \mid \mu(\underline{\pi})>\mu^{*}\right\},
$$

with $\mu^{*}$ given by (23).

This means that, whenever some cash flows $\alpha_{i}$ are negative, the optimization procedures described in the following paragraphs will not always take into account all possible investment portfolios. However, restricting optimization to $\Theta$ is intuitive, as in most situations it will be desirable to obtain a portfolio for which the expected value of the future available assets is positive at any time.

Maximizing the probability level for a given target wealth For a given investment portfolio $\underline{\pi}$, the probability that the final wealth $W_{n}(\underline{\pi})$ exceeds some positive value $x$ is given by

$$
p=\operatorname{Pr}\left[W_{n}(\underline{\pi})>x\right]=1-\mathrm{F}_{W_{n}(\underline{\pi})}(x)=\overline{\mathrm{F}}_{W_{n}(\underline{\pi})}(x) .
$$

We determine the portfolio $\underline{\pi}^{*}$, for which the probability $p$ of reaching at least a certain amount $x$ at time $n$ is maximal. Denoting this probability by $p^{*}$, we have:

$$
p^{*}=\max _{\underline{\pi} \in \Theta} \overline{\mathrm{F}}_{W_{n}(\underline{\pi})}(x),
$$

with $\Theta$ given by (35). Consider two portfolios, $\underline{\pi}_{1}$ and $\underline{\pi}_{2}$, with $\sigma\left(\underline{\pi}_{1}\right)=\sigma\left(\underline{\pi}_{2}\right)$ and $\mu\left(\underline{\pi}_{1}\right)<\mu\left(\underline{\pi}_{2}\right)$. As shown in Dhaene et al. (2005), we have that $\overline{\mathrm{F}}_{W_{n}\left(\underline{\pi}_{1}\right)}(x) \leq \overline{\mathrm{F}}_{W_{n}\left(\underline{\pi}_{2}\right)}(x)$. Therefore the solution of (37) is to be found on the Capital Market Line, which means we can replace (37) by

$$
p^{*}=\max _{\sigma \in \Theta^{\prime}} \overline{\mathrm{F}}_{W_{n}\left(\underline{\pi}^{\sigma}\right)}(x),
$$

with

$$
\Theta^{\prime}=\left\{\sigma \mid \underline{\pi}^{\sigma} \in \Theta\right\}
$$


Recall that, for a given $\sigma$, the corresponding mean-variance efficient portfolio $\underline{\pi}^{\sigma}$ is given by

$$
\underline{\pi}^{\sigma}=\sigma \frac{\Sigma^{-1} \cdot(\underline{\mu}-r \underline{1})}{\sqrt{(\underline{\mu}-r \underline{1})^{T} \cdot \Sigma^{-1} \cdot(\underline{\mu}-r \underline{1})}} .
$$

Finally, we approximate the optimal investment strategy $\underline{\pi}^{*}$ by $\underline{\pi}^{l}$, using the comonotonic lower bound approximation $W_{n}^{l}(\underline{\pi})$. This results in the approximated optimal probability level $p^{l}$ :

$$
p^{l}=\max _{\sigma \in \Theta^{\prime}} \overline{\mathrm{F}}_{W_{n}^{l}\left(\underline{\pi}^{\sigma}\right)}(x) \approx p^{*}
$$

Maximizing the target capital for a given probability level For a given investment strategy $\underline{\pi}$, we define the $p$-target capital $K$ as the $(1-p)$-th order quantile of the final wealth:

$$
K=Q_{1-p}\left[W_{n}(\underline{\pi})\right] .
$$

This target capital can be interpreted as the maximal amount that will be available at time $n$, with a probability of at least $p$. We determine the optimal portfolio $\underline{\pi}^{*}$, by maximizing the target capital that can be reached for a given probability level $p$. We have

$$
K^{*}=\max _{\underline{\pi} \in \Theta} Q_{1-p}\left[W_{n}(\underline{\pi})\right]
$$

with $\Theta$ given by (35).

As shown in Dhaene et al. (2005), the solution of (43) is to be found on the Capital Market Line, implying we can replace (43) by

$$
K^{*}=\max _{\sigma \in \Theta^{\prime}} Q_{1-p}\left[W_{n}\left(\underline{\pi}^{\sigma}\right)\right],
$$

with $\Theta^{\prime}$ and $\underline{\pi}^{\sigma}$ given by (39) and (40) respectively.

As in Section 2.2.2, we approximate the optimal investment strategy $\underline{\pi}^{*}$ by $\underline{\pi}^{l}$, using the comonotonic lower bound approximation $W_{n}^{l}(\underline{\pi})$. This results in the approximated maximal target capital $K^{l}$ :

$$
K^{l}=\max _{\sigma \in \Theta^{\prime}} Q_{1-p}\left[W_{n}^{l}\left(\underline{\pi}^{\sigma}\right)\right] \approx K^{*}
$$

Numerical Illustration Suppose $n=31, \alpha_{i}=10$ if $i$ is not a multiple of 5 , and $\alpha_{5 k}=$ -45 for $k=1, \ldots, 6$. Furthermore, suppose there are three risky asset classes available, with drift vector $\underline{\mu}^{T}=(0.02,0.05,0.075)$, standard deviations $\underline{\sigma}^{T}=(0.01,0.1,0.18)$ and correlations $\rho_{1,2}=-0.10, \rho_{1,3}=0.03$ and $\rho_{2,3}=0.50$. Determining the set of admissable portfolios $\Theta$ leads to:

$$
\Theta=\left\{\underline{\pi} \mid E\left[V_{i}(\underline{\pi})\right]>0 ; i=1, \ldots, n-1\right\}=\{\underline{\pi} \mid \mu(\underline{\pi}) \geq 0.0242\}
$$


Hence we can use the comonotonic lower bound approximations for all portfolios with drift bigger than $2.42 \%$. As a first application, assume a target wealth $x$ equal to 0 . Using our lower bound approximation leads to a maximal survival probability

$$
p^{l}=\max _{\underline{\pi} \in \Theta} \overline{\mathrm{F}}_{W_{n}^{l}\left(\underline{\pi}^{\sigma}\right)}(0)=0.87=(0.9954)^{30},
$$

with the corresponding optimal investment strategy $\underline{\pi}^{l}$ equal to $\left(\underline{\pi}^{l}\right)^{T}=$ $(0.1808,0.5167,0.3025)$, with drift $\mu\left(\underline{\pi}^{l}\right)=0.0521$ and standard deviation $\sigma\left(\underline{\pi}^{l}\right)=0.0920$. Note that this optimal strategy indeed satisfies condition (46).

As a second application we compute the maximal target wealth $K^{l}$ and corresponding optimal investment strategy $\underline{\pi}^{l}$ for a range of probability levels $p$. The results are given in Table 3.

Note that for $p=0.90$ en $p=0.95$ we find $K^{l}=0$. Since we are maximizing the quantiles of $W_{n}^{l}=\max \left(V_{n}^{l}, 0\right)$, this result means that we can not reach a positive target wealth with the given probabilities. Hence the maximal surplus $V_{n}^{l}$ that can be reached is negative. In fact, as can be seen from (47), it is not possible to find a strategy leading to a positive surplus for any probability level above 0.87 .

\begin{tabular}{c|cccccc} 
& \multicolumn{7}{|c}{$p$} & & & \\
& $70 \%$ & $75 \%$ & $80 \%$ & $85 \%$ & $90 \%$ & $95 \%$ \\
\hline$\pi_{1}^{l}$ & $0.00 \%$ & $0.00 \%$ & $0.00 \%$ & $5.54 \%$ & $43.76 \%$ & $80.75 \%$ \\
$\pi_{2}^{l}$ & $45.82 \%$ & $53.07 \%$ & $58.05 \%$ & $59.51 \%$ & $35.88 \%$ & $13.14 \%$ \\
$\pi_{3}^{l}$ & $54.18 \%$ & $46.93 \%$ & $41.95 \%$ & $34.95 \%$ & $20.36 \%$ & $6.11 \%$ \\
$\mu\left(\underline{\pi}^{l}\right)$ & $6.35 \%$ & $6.17 \%$ & $6.05 \%$ & $5.71 \%$ & $4.20 \%$ & $2.73 \%$ \\
$\sigma\left(\underline{\pi}^{l}\right)$ & $12.68 \%$ & $12.01 \%$ & $11.60 \%$ & $10.60 \%$ & $6.28 \%$ & $2.21 \%$ \\
$K^{l}$ & 27.73 & 19.40 & 11.54 & 3.84 & 0.00 & 0.00
\end{tabular}

Table 3: Maximal target wealth $K^{l}$ and optimal strategy $\pi^{l}$ for survival probabilities $p$.

\subsection{Reserves for future obligations}

In this section we discuss the reserving problem, which is in some sense the dual problem of the terminal wealth problem as described in Section 2.2. Consider a series of deterministic obligations $\alpha_{1}, \ldots, \alpha_{n}$, due at time $1, \ldots, n$ respectively. Being able to meet these obligations requires appropriate funding, meaning that a reserve has to be set up at time 0 . In Dhaene et al. (2005) the reserving problem is discussed in detail, with the restriction that all obligations $\alpha_{i}$ are positive. It will follow from our main result that $\alpha_{n}$ has to be positive. However, $\alpha_{i}$ for $i=1, \ldots, n-1$ can take any value, positive or negative. A negative obligation can be interpreted as adding an amount to the reserve on the account.

As explained in Dhaene et al. (2005), the variables of interest in this setting, of which we would like to describe the distribution, are the so-called stochastic future obligations. 
These random variables $R_{k}$ are given by:

$$
R_{k}=\sum_{i=k+1}^{n} \alpha_{i} e^{Z_{i}}, \quad k=0, \cdots, n-1
$$

with $Z_{i}=-\sum_{j=k+1}^{i} Y_{j}$, for $i=k+1, \ldots, n$. Note that $R_{k}$ in (48) has the same general form as (2). For a given $k, R_{k}$ is the stochastically discounted value at time $k$ of all future obligations from time $k$ on.

Our goal is to approximate the distribution function of $R_{0}$. As for the surplus in the previous section, we see that $R_{0}$ can become negative. To avoid this we only look at the stochastic provision $S_{0}$ available at time 0 , which we define as:

$$
S_{0}=\max \left[R_{0}, 0\right]
$$

As explained in Section 2.1, we focus on the lower bound approximation, which we denote as $R_{0}^{l}$ and $S_{0}^{l}$ respectively. The amount of money that has to be set aside to meet future liabilities can then be determined by applying an appropriate distortion risk measure to this approximation.

As derived in Dhaene et al. (2005), the optimal conditioning random variable $\Lambda$ of the form (6) has in this setting coefficients $\beta_{j}$ given by:

$$
\beta_{j}=-\sum_{k=j}^{n} \alpha_{k} e^{k\left(-\mu+\sigma^{2}\right)}
$$

for $j=1, \ldots n$. This leads to the following lower bound approximation $R_{0}^{l}$ :

$$
R_{0}^{l} \stackrel{d}{=} \sum_{i=1}^{n} \alpha_{i} e^{-i \mu+\left(1-\frac{1}{2} r_{i}^{2}\right) i \sigma^{2}+r_{i} \sigma \sqrt{i} \Phi^{-1}(U)},
$$

where $\stackrel{d}{=}$ stands for 'equality in distribution', $U$ is uniformly distributed on $(0,1)$, and the coefficients $r_{i}$ are given by

$$
r_{i}=\frac{-\sum_{j=1}^{i} \beta_{j}}{\sqrt{i} \sqrt{\sum_{j=1}^{n} \beta_{j}^{2}}}, \quad i=0, \cdots, n .
$$

Throughout the remainder of this section we use the notation $f$ to denote the function

$$
f(p)=\sum_{i=1}^{n} \alpha_{i} e^{-i \mu+\left(1-\frac{1}{2} r_{i}^{2}\right) i \sigma^{2}+r_{i} \sigma \sqrt{i} \Phi^{-1}(p)},
$$

with $p \in(0,1)$. Combining (49) and (51) we see that $S_{0}^{l} \stackrel{d}{=} \max [f(U), 0]$.

In order to proof the main result of this section, we state the following Lemma, which is similar to Lemma 1: 
Lemma 2 Let $f(p)$ be defined by (53) and $\beta_{j}$ by (50). If $\beta_{j}<0$ for $j=1,2, \ldots, n$, then, for any $p$ in the unit interval $(0,1), f(p) \geq 0$ implies $f^{\prime}(p)>0$.

Proof. This proof is analogous to the proof of Lemma 1 (see Vanduffel et al. (2005)). Since $\beta_{j}<0$ for $j=1,2, \ldots, n$ we see from $(52)$ that $r_{i}>0$ for $i=0, \ldots, n$. Furthermore we see that

$$
r_{i} \sigma_{Z_{i}}=-\sigma \frac{\sum_{j=1}^{i} \beta_{j}}{\sqrt{\sum_{j=1}^{n} \beta_{j}^{2}}}, \quad i=0, \cdots, n,
$$

since $\sigma_{Z_{i}}=\sqrt{i} \sigma$. Hence the sequence $\left\{r_{i} \sigma_{Z_{i}}\right\}_{0 \leq i \leq n}$ is strictly decreasing and strictly positive.

From (53) we find, by application of the chain rule, that

$$
f^{\prime}(p)=\frac{1}{\Phi^{\prime}\left[\Phi^{-1}(p)\right]} \sum_{i=1}^{n} a_{i} e^{-i \mu+\left(1-\frac{1}{2} r_{i}^{2}\right) i \sigma^{2}+r_{i} \sigma \sqrt{i} \Phi^{-1}(p)} r_{i} \sigma_{Z_{i}} .
$$

Assume $f(p) \geq 0$ for some $p$ in the unit interval. Since also $\frac{1}{\Phi^{\prime}\left[\Phi^{-1}(p)\right]}>0$ we find that

$$
\begin{aligned}
f^{\prime}(p) & >\frac{r_{1} \sigma}{\Phi^{\prime}\left[\Phi^{-1}(p)\right]} \sum_{i=1}^{n} a_{i} e^{-i \mu+\left(1-\frac{1}{2} r_{i}^{2}\right) i \sigma^{2}+r_{i} \sigma \sqrt{i} \Phi^{-1}(p)} \\
& =\frac{r_{1} \sigma}{\Phi^{\prime}\left[\Phi^{-1}(p)\right]} f(p) \geq 0,
\end{aligned}
$$

which completes the proof.

The main result of this section is stated in the following Theorem.

Theorem 2 If the conditioning random variable $\Lambda$ is chosen as (6) with coefficients (50), and if

$$
E\left[R_{j}\right]>0, \quad j=0, \cdots, n-1,
$$

then the quantiles of $S_{0}^{l}$ are given by

$$
Q_{p}\left[S_{0}^{l}\right]=\max [f(p), 0] \quad 0<p<1,
$$

whereas the distribution function of $S_{0}^{l}$ follows from

$$
f\left(F_{S_{0}^{l}}(x)\right)=x, \quad x \geq 0
$$

with $f(p)$ defined by (53).

Proof. This proof is analogous to the reasoning in Section 3 of Vanduffel et al. (2005a). It follows from (50) that for $j=1,2, \ldots, n$,

$$
\beta_{j}=-e^{-(j-1)\left(-\mu+\sigma^{2}\right)} \mathrm{E}\left[R_{j-1}\right] .
$$


In other words, condition (54) implies that $\beta_{j}<0$ for $j=1,2, \ldots, n$. It is easy to show that this implies

$$
\lim _{p \rightarrow 0} f(p)=0 \text { and } \lim _{p \rightarrow 1} f(p)=+\infty .
$$

We find from Lemma 2 that the function $\max [f(p), 0]$ is non-decreasing (and continuous) on the interval $(0,1)$. Using $S_{0}^{l} \stackrel{d}{=} \max [f(U), 0]$ and the fact that $Q_{p}(g(X))=g\left(Q_{p}[X]\right)$ and $Q_{p}^{+}[g(X)]=g\left(Q_{p}^{+}[X]\right)$ for any non-decreasing function $g$ and any $p \in(0,1)$, we see that the quantiles of $S_{0}^{l}$ can easily be determined analytically in this case:

$$
Q_{p}\left[S_{0}^{l}\right]=Q_{p}\left[S_{0}^{l}\right]=\max [f(p), 0], \quad p \in(0,1) .
$$

It is easy to show that

$$
F_{S_{0}^{l}}(x)=\sup \{p \in(0,1) \mid f(p) \leq x\} .
$$

Using this last equation (60), together with (58), Lemma 2 and the fact that $f$ is continuous on $(0,1)$, we see that the d.f. of $S_{0}^{l}$ can be determined from

$$
f\left(F_{S_{0}^{l}}(x)\right)=x, \quad x \geq 0
$$

This completes the proof.

It is clear that conditions (54) will be satisfied in a lot of practical situations. When working in a provisioning context, it is natural to suppose that the expected present value of future obligations will be positive at any time.

For given cash flows $\alpha_{i}$, we have $\mathrm{E}\left[R_{j}\right]=\sum_{k=j+1}^{n} \alpha_{k} e^{(k-j)\left(-\mu+\sigma^{2}\right)}$. This function depends on both $\mu$ and $\sigma$. Moreover, it is decreasing in $\mu$, and increasing in $\sigma$, which means that, in contrast to the previous section (see (23)), it is not useful to rewrite conditions (54) as a single condition.

We have shown that allowing some of the obligations to be negative does not necessarily mean that comonotonic lower bound approximations can not be used to approximate the distribution function of the initial provision. This is important, since it again significantly improves the practical applicability of the approximations.

There are plenty of practical examples where negative future obligations occur. For instance in case of a life insurance, premiums will typically exceed benefit payments in the first years, whereas the majority of the benefits are paid near the end of the contract. For these contracts conditions (54) will typically be satisfied, since most negative obligations will occur in the near future, meaning that the lower bound approximation can be used to compute an initial provision.

Deriving formulas for the special case of constant future obligations, and applying the results of this section to optimal portfolio selection is completely analogous to Section 2.2.1 and Section 2.2.2 respectively. We do not discuss this here. 


\section{Minimal Return Requirement}

In this section we generalize the problem of finding an optimal constant mix investment strategy in a provisioning or savings context to the case where a minimal return requirement is imposed. Assume the owner of the invested capital requires a minimal yearly return of $r$. This minimal level of the return should be obtained with a given (high) probability of at least $(1-\varepsilon)$ over each period of $m$ years.

Note that $r$ is not necessarily strictly positive. A capital guarantee requirement for example corresponds to a minimal return of 0 . Also, a negative $r$ can be used to ensure the loss of capital can not exceed a specified amount.

Using notations as defined in Section 1.1, an amount of 1 invested according to a strategy $\underline{\pi}$ will grow to the random amount $e^{Y_{1}(\underline{\pi})+\ldots+Y_{m}(\underline{\pi})}$ after a period of $m$ years. Hence, the minimal return requirement can be expressed as

$$
\operatorname{Pr}\left[e^{Y_{1}(\underline{\pi})+\ldots+Y_{m}(\underline{\pi})} \geq e^{m r}\right] \geq 1-\varepsilon,
$$

or, equivalently,

$$
m r \leq F_{Y_{1}(\underline{\pi})+\ldots+Y_{m}(\underline{\pi})}^{-1}(\varepsilon) .
$$

The distribution function of the random variable $Y_{1}(\underline{\pi})+\ldots+Y_{m}(\underline{\pi})$ is characterized by

$$
Y_{1}(\underline{\pi})+\ldots+Y_{m}(\underline{\pi}) \stackrel{d}{=} m\left(\mu(\underline{\pi})-\frac{1}{2} \sigma^{2}(\underline{\pi})\right)+\sqrt{m} \sigma(\underline{\pi}) \Phi^{-1}(U),
$$

with $U$ uniformly distributed on the unit interval, and $\Phi$ the standard normal cdf. This implies that the $\varepsilon$-quantile of $Y_{1}(\underline{\pi})+\ldots+Y_{m}(\underline{\pi})$ is given by

$$
F_{Y_{1}(\underline{\pi})+\ldots+Y_{m}(\underline{\pi})}^{-1}(\varepsilon)=m\left(\mu(\underline{\pi})-\frac{1}{2} \sigma^{2}(\underline{\pi})\right)+\sqrt{m} \sigma(\underline{\pi}) \Phi^{-1}(\varepsilon) .
$$

Using (65), the return guarantee requirement (63) can be rewritten as

$$
\mu(\underline{\pi})-\frac{1}{2} \sigma^{2}(\underline{\pi}) \geq r+\frac{1}{\sqrt{m}} \sigma(\underline{\pi}) \Phi^{-1}(1-\varepsilon),
$$

or, equivalently,

$$
\mathrm{E}\left[Y_{i}(\underline{\pi})\right] \geq r+\sqrt{\frac{\operatorname{Var}\left[Y_{i}(\underline{\pi})\right]}{m}} \Phi^{-1}(1-\varepsilon) .
$$

This condition is in accordance with intuition, as the right hand side of inequality (67) is increasing for respectively increasing $r$, decreasing $m$ and decreasing $\varepsilon$.

In the framework of optimal portfolio selection, condition (67) can be used as a constraint on the admissable investment portfolios $\underline{\pi}$, in order to guarantee a minimal yearly return $r$, with a related probability level $(1-\varepsilon)$ over each period of $m$ years. 


\subsection{Numerical Illustration}

Using notations as in Dhaene et al. (2005) and Section 1.1, suppose $n=30$, and $\alpha_{i}=10$ for $i=0, \ldots, 29$. Furthermore, suppose we have the three asset classes available which were described in Section 2.2.2. The minimal return requirement (67) can be applied both in a context of saving and provisioning. Here we work in the former framework.

The terminal wealth, or the amount of money available on our account at time $n$, is equal to:

$$
W_{n}(\underline{\pi})=\sum_{i=0}^{n-1} \alpha_{i} e^{\sum_{j=i+1}^{n} Y_{j}(\underline{\pi})} .
$$

We approximate $W_{n}(\underline{\pi})$ by the comonotonic lower bound approximation $W_{n}^{l}(\underline{\pi})$, as defined in Dhaene et al. (2005):

$$
W_{n}^{l}(\underline{\pi})=\sum_{i=0}^{n-1} \alpha_{i} e^{(n-i) \mu(\underline{\pi})-\frac{1}{2} \sigma^{2}(\underline{\pi})+r_{i}(\underline{\pi}) \sqrt{n-i} \sigma(\underline{\pi}) \Phi^{-1}(U)}
$$

We want to determine the strategy $\underline{\pi}^{*}$ leading to a maximal terminal wealth $K^{*}$ :

$$
K^{*}=\max _{\underline{\pi}} F_{W_{n}(\underline{\pi})}^{-1}(1-p) .
$$

By maximizing the quantiles of the comonotonic lower bound approximation (69) we can approximate $\underline{\pi}^{*}$ and $K^{*}$ as:

$$
K^{l}=\max _{\underline{\pi}} F_{W_{n}^{l}(\underline{\pi})}^{-1}(1-p) \approx K^{*} .
$$

Denote the approximated optimal strategy leading to $K^{l}$ as $\underline{\pi}^{l}$. In our example we use a certainty level $p=0.85$. In Table 4 the influence of a return requirement on the optimal investment portfolio is illustrated. If we do not impose a return requirement, we find as a result an optimal strategy $\underline{\pi}^{l}=(0,0.5611,0.4389)^{T}$, with corresponding drift $\mu\left(\underline{\pi}^{l}\right)=0.0610$ and standard deviation $\sigma\left(\underline{\pi}^{l}\right)=0.1176$. The maximized terminal wealth $K^{l}$ amounts to 499.72 .

\begin{tabular}{c|ccc} 
& $\begin{array}{c}\text { no return } \\
\text { requirement }\end{array}$ & $\begin{array}{c}\text { capital guarantee } \\
(0 \%)\end{array}$ & $\begin{array}{c}\text { positive return } \\
\text { requirement }(1 \%)\end{array}$ \\
\hline$\pi_{1}^{l}$ & $0 \%$ & $17.57 \%$ & $54.33 \%$ \\
$\pi_{2}^{l}$ & $56.11 \%$ & $52.05 \%$ & $29.40 \%$ \\
$\pi_{3}^{l}$ & $43.89 \%$ & $30.38 \%$ & $16.72 \%$ \\
$\mu\left(\underline{\pi}^{l}\right)$ & $6.10 \%$ & $5.23 \%$ & $3.78 \%$ \\
$\sigma\left(\underline{\pi}^{l}\right)$ & $11.76 \%$ & $9.24 \%$ & $5.09 \%$ \\
$K^{l}$ & 499.72 & 489.0 & 460.4
\end{tabular}

Table 4: Influence of return requirement on optimal investment strategy.

Now suppose we use formula (67) as a constraint on the admissable investment portfolios, with parameters $r=0, m=10$ and $\epsilon=0.05$. In other words, we require a capital 
guarantee, with a return at least equal to zero, over each period of 10 years, with a probability of $95 \%$. As can be seen from Table 4, imposing this leads to an optimal strategy $\underline{\pi}^{l}=(0.1757,0.5205,0.3038)^{T}$, with $\mu\left(\underline{\pi}^{l}\right)=0.0523$ and $\sigma\left(\underline{\pi}^{l}\right)=0.0924$. The maximized terminal wealth $K^{l}$ in this case amounts to 489.0. As a second example, suppose $m=10$ and $\epsilon=0.05$, but we require a positive return of at least $1 \%$, or $r=0.01$. This leads to $\underline{\pi}^{l}=(0.5433,0.2940,0.1672)^{T}$, with $\mu\left(\underline{\pi}^{l}\right)=0.0378, \sigma\left(\underline{\pi}^{l}\right)=0.0509$ and $K^{l}=460.36$.

From these results we can conclude that imposing a return requirement leads to a more conservative optimal portfolio, with higher proportions invested in less risky assets, and hence with a lower drift and volatility. Also, the resulting maximal terminal wealth is significantly lower. These results are in correspondence with intuition.

\section{Conclusion}

In this paper we analyzed several applications of optimal portfolio selection problems. We extended some of the results obtained by Dhaene et al. (2005) and Vanduffel et al. (2005a), expanding the scope of problems to which comonotonic approximations can be applied.

First we investigated if the lower bound approximations based on comonotonicity (see e.g. Dhaene et al. $(2002 \mathrm{a}, \mathrm{b}))$ are still valid in case of cash flows with fluctuating signs. In the context of saving and terminal wealth we showed that the lower bound approximation works perfectly as long as the expected surplus remains positive at any time in the future. Similarly we showed that, when working in a context of reserving, the approximation is valid in case the expected future obligations remain positive. In both cases these conditions are intuitive, and satisfied in most practical situations. We also pointed out how these problems can readily be applied to optimal portfolio selection, illustrating it with numerical examples.

Next we explained how a minimal return guarantee can be introduced in our optimal portfolio selection framework. We obtained a basic, but very useful and intuitively clear formula, and illustrated the effect of such a guarantee numerically.

Overall this paper gives several extensions to comonotonic approximations, and its application to optimal portfolio selection. This significantly increases the practical applicability of the main results obtained e.g. in Dhaene et al. (2005). Future research would consist in generalizing some of these results even further, for example to the case of stochastic cash flows, or an elliptically distributed return process. Also, the problem of liabilities with changing signs still has to be solved for more general (distortion) risk measures, such as TVaR or CTE.

Acknowledgement 1 The authors acknowledge the financial support by the Onderzoeksfonds K.U.Leuven (GOA/0\%: Risk Modeling and Valuation of Insurance and Financial Cash Flows, with Applications to Pricing, Provisioning and Solvency). 


\section{References}

[1] Asmussen, S., Royas-Nandayapa (2005). "Sums of dependent lognormal random variables: asymptotics and simulation", Stochastic Series at Department of Mathematical Sciences, University of Aarhus, Research Report number 469.

[2] Björk, T. (1998). "Arbitrage Theory in Continuous Time", Oxford University Press, pp. 311.

[3] Black, F., Scholes, M. (1973). "The pricing of options and corporate liabilities", Journal of Political Economy, 81 (May-June), 637-659.

[4] Cesari, R., Cremonini, D. (2003). "Benchmarking, portfolio insurance and technical analysis: a Monte Carlo comparison of dynamic strategies of asset allocation", Journal of Economic Dynamics and Control, 27, 987-1011.

[5] Denuit, M., Dhaene, J., Goovaerts, M.J., Kaas, R. (2005). "Actuarial theory for dependent risks: measures, orders and models," Wiley, pp. 440.

[6] Dhaene, J., Denuit, M., Goovaerts, M.J., Kaas, R. \& Vyncke, D. (2002a). "The concept of comonotonicity in actuarial science and finance: Theory," Insurance: Mathematics and Economics 31(1), 3-33.

[7] Dhaene, J., Denuit, M., Goovaerts, M.J., Kaas, R. \& Vyncke, D. (2002b). "The concept of comonotonicity in actuarial science and finance: Applications," Insurance: Mathematics and Economics 31(2), 133-161.

[8] Dhaene, J., Vanduffel, S., Goovaerts, M.J., Kaas, R. \& Vyncke, D. (2005). "Comonotonic approximations for optimal portfolio selection problems," Journal of Risk and Insurance 72 (2), 253-301.

[9] Dhaene, J., Vanduffel, S., Tang, Q., Goovaerts, M.J., Kaas, R. \& Vyncke, D. (2006). "Risk measures and comonotonicity: a review," Stochastic Models, 22, 573-606.

[10] Dufresne, D. (2004). "The log-normal approximation in financial and other computations," Advances in Applied Probability 36: 747-773.

[11] Huang, H., Milevsky, M.A., Wang, J. (2004). "Ruined Moments in Your Life: How Good Are the Approximations?", Insurance: Mathematics and Economics 34(3): 421-447.

[12] Kaas, R., Dhaene, J. \& Goovaerts, M.J. (2000). "Upper and lower bounds for sums of random variables," Insurance: Mathematics and Economics 27, 151-168.

[13] Kaas, R., Goovaerts, M.J., Dhaene, J. \& Denuit, M. (2008). "Modern Actuarial Risk Theory, Using R," Springer, pp 381.

[14] Levy, H. (2004). "Asset Return Distributions and the Investment Horizon," The Journal of Portfolio Management, Spring 2004: 47-62. 
[15] Merton, R. (1971). "Optimum consumption and portfolio rules in a continuous-time model," Journal of Economic Theory, 3, 373-413.

[16] Milevsky, M.A., Posner, S. (1998). "Asian Options, the Sum of Lognormals, and the Reciprocal Gamma Distribution," Journal of Financial and Quantitative Analysis 33(3): 409-422.

[17] Milevsky, M.A., Robinson, C. (2000). "Self-Annuitization and Ruin in Retirement," North American Actuarial Journal 4(4): 112-124.

[18] Vanduffel, S., Dhaene, J. \& Goovaerts, M.J. (2005a). "On the evaluation of savingconsumption plans," Journal of Pension Economics and Finance, 4(1), 17-30.

[19] Vanduffel, S., Hoedemakers, T., Dhaene, J. (2005b). "Comparing approximations for risk measures of sums of non-independent lognormal random variables," North American Actuarial Journal, vol. 9(4), 71-82. 